\title{
Bloqueos locoregionales anestésicos en urgencias de traumatología. WALANT Surgery
}

Ortega Yago 1,2, Amparo; BaixauliPerelló, Emilio²

En traumatología cada vez está más extendido el uso de técnicas de anestesia loco regionales, tanto para la urgencia traumatológica como también para cirugías de miembro superior ${ }^{7-3}$ e inferior ${ }^{4-6}$.

Mediante el aprendizaje de estas técnicas, se puede llevar a cabo, y sin necesidad de realizar una anestesia general, diversos procedimientos que facilitan una mayor recuperación del paciente e incluso su colaboración mientras se está realizando la intervención, gracias a la técnicaWalant (Wide awake, local, anesthesia, no tourniquet)

Para ello, será necesario un buen conocimiento tanto de la anatomía, como de las características de los anestésicos y de los coadyuvantes que utilizaremos para realizar los bloqueos locorregionales.

${ }^{1}$ Corresponding autor: Amparo Ortega Yago. La Fe Hospital. ortegayago94@gmail.com 2 La Fe Hospital, Av Fernando abril martorell 106, Valencia Spain 


\section{INTRODUCIENDO EL WALANT}

Una técnica que cada vez está en mayor auge es el WALANT. El WALANT hace referencia al "wide, awake, local, anesthesia, no tourniquet", es decir, en la intervención el paciente está despierto, realizado con anestesia local y sin torniquete.

Las ventajas del Walant es que es una técnica que no requiere manguito de isquemia, y por tanto el cirujano no se ve limitado por el tiempo de la misma. Entre otras ventajas se encuentra que el paciente puede colaborar en la intervención quirúrgica, y que no requiere vía intravenosa ni monitorización dado que la anestesia es de tipo local.

\section{ANESTÉSICOS LOCALES}

Los anestésicos que se usan en esta técnica son muy variados. Clásicamente se han dividido entre ésteres (no usados prácticamente) y amidas, siendo que en traumatología se usa la lidocaína, con un inicio de acción más rápido, y la mepivacaína, con un inicio de acción más lenta pero la más segura a nivel cardiovascular. Otra de las virtudes de la mepivacaína es que produce menos vasodilatación que la lidocaína.

Sobre la toxicidad de los $\mathrm{AL}$, cabe destacar que puede producirse a nivel local sobre el nervio, o bien puede ser sistémica (sea por sobredosis o por inyección intravascular) ${ }^{6}$. El primer síntoma que el paciente nos referirá y que nos debe alertar de que el anestésico ha pasado a la circulación es que refiera sabor metálico de la lengua y boca. Entre otros posibles efectos entra el mareo, y a nivel cardiovascular con mayores dosis hipertensión y taquicardia.

\section{COADYUVANTES}

¿Qué sucede con los coadyuvantes para la anestesia?

\section{ADRENALINA}

Una duda que suele surgir al cirujano es si puede hacer uso de la adrenalina junto con la anestesia local. Clásicamente se ha transmitido durante generaciones que la adrenalina junto con el anestésico local produce necrosis a nivel distal.

Esta mala fama viene porque antes de 1950 se utilizaba la procaína como anestésico local. La alarma saltó en 1948, cuando se dieron cuenta que al administrar procaína sin adrenalina- producía necrosis tisular. Esto se explica porque la procaína tiene un $\mathrm{pH}$ mucho menor que la lidocaína (pudiendo llegar incluso a menos de 1) y también porque a veces utilizaban botes de procaína caducados porque la ley no obligaba a registrar fechas de caducidad en anestésicos locales. ${ }^{8}$

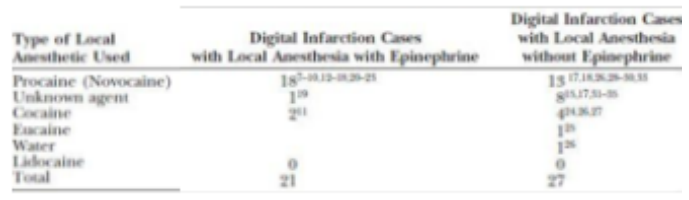

Tabla 1: Se observa que la lidocaína con y sin adrenalina tiene mucho menor riesgo comparado con otros anestésicos locales de producir infartos a nivel distal. ${ }^{8}$

Recientemente la Cochrane ha publicado una revisión sistemática donde no encuentra evidencia a favor o en contra del uso de adrenalina con anestesia local ${ }^{9}$. Sin embargo, cada vez está aumentando la evidencia de que usar adrenalina con anestésicos locales en zonas distales es segura ${ }^{10-13}$ 
Normalmente las proporciones suelen ser de 1:10.000. Hoy en día existen múltiples combinaciones, como la articaína y la adrenalina.

Existe un antídoto llamado fentolamina. Este fármaco es un alfa bloqueante, y revierte el efecto de la adrenalina en aproximadamente 80 minutos. La relación de aplicación de la fentolamina es de 1:1 con respecto a la adrenalina. ${ }^{14}$

Aun así, se suele intentar evitar el uso de la adrenalina en aquellos pacientes con enfermedades cardiovasculares $O$ que tomen fármacos como los IMAOs

\section{BICARBONATO}

Algunos anestésicos locales tienen tendencia a precipitar con facilidad, lo cual producen dolor al paciente. Es por ello que El uso del bicarbonato al $8.4 \%$ en proporción 10:1 (lidocaína:bicarbonato) está ampliamente establecido para disminuir el dolor de la inyección en los pacientes ${ }^{15,16}$

\section{CASOS ESPECIALES}

\section{EMBARAZADAS Y MUJERES DANDO LACTANCIA}

\section{Lidocaína}

El uso de lidocaína es de categoría B en las mujeres embarazadas. Por tanto, puede ser utilizado si el beneficio es mayor que el riesgo. ${ }^{17}$

La lidocaína es excretada por leche materna en pequeñas dosis. Hay que tener precaución a la hora de utilizarlo, aunque los efectos secundarios en el lactante serían raros y estarían relacionados con reacciones alérgicas o de idiosincrasia. ${ }^{17}$

\section{Mepivacaína}

El uso de lidocaína es de categoría $C$ en las mujeres embarazadas. Por tanto, sólo debería utilizado si el beneficio es mucho mayor que el riesgo. ${ }^{18}$

No se sabe si la mepivacaína es excretada por la leche, por ello hay que administrarla con cautela. ${ }^{18}$

\section{EDAD PEDIÁTRICA}

\section{Lidocaína}

Puede ser usada con precaución ajustándose las dosis al peso del paciente. 17

\section{Mepivacaína}

Puede ser usada con precaución ajustándose las dosis al peso del paciente. 18

\section{BLOQUEOS LOCO-REGIONALES EN TRAUMATOLOGÍA}

EXTREMIDAD SUPERIOR

La extremidad superior es el sitio más habitual donde se hace el bloqueo locoregional, tanto en urgencias de traumatología como también en la cirugía.

Los principales bloqueos en traumatología son:

\section{Bloqueo ramas terminales de muñeca}

\section{Radial}

- Qué se anestesia: Dorso de la mano (primeros dedos + borde radial del 4)

- Cómo se anestesia: Buscar la estiloides radial y la arteria radial. Se inyecta anestésico, y se mueve posteriormente la aguja por el borde radial hasta la cara dorsal de la estiloides.

El nervio radial suele requerir infiltración en distintos puntos para 
cubrir de forma adecuada el territorio de la curvatura alrededor de la muñeca.

Muchas veces requiere más anestésico de lo normal (>10 ml) Este nervio es superficial, por tanto, no requiere infiltración excesivamente profunda.

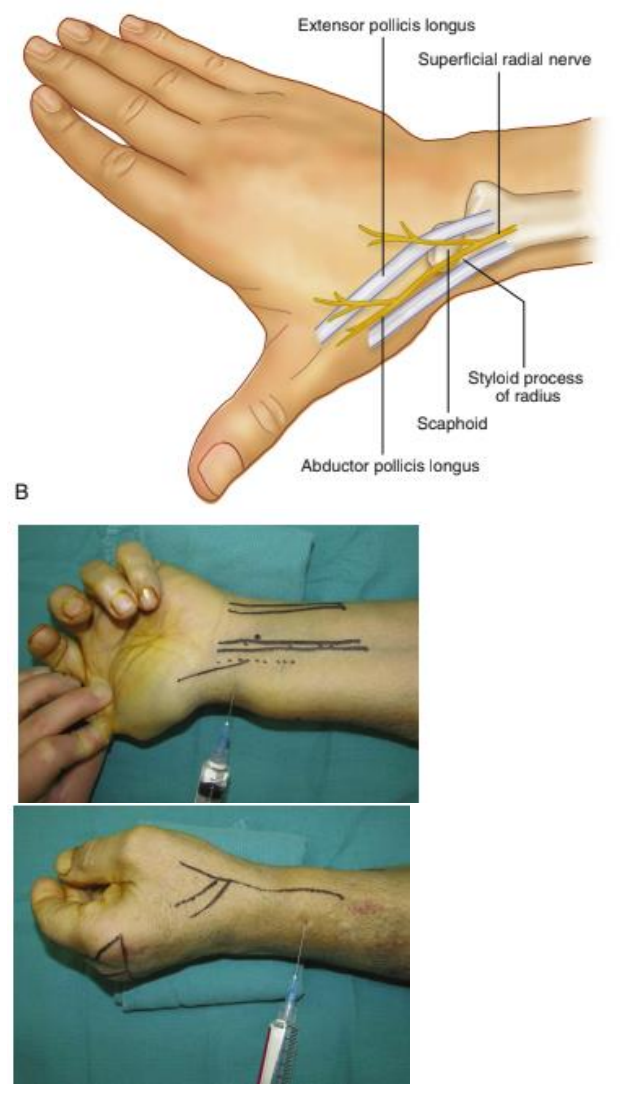

Imagen 1: localización del nervio radial y anestesia del mismo. ${ }^{19}$

\section{Cubital}

- Qué se anestesia: Se anestesia borde cubital 4 dedo y 5 dedo en cara dorsal y volar.

- Cómo se anestesia: Muñeca en hiperextensión. Buscar pulso cubital y tendón flexor cubital del carpo. Inyectar en el borde cubital del FCU.

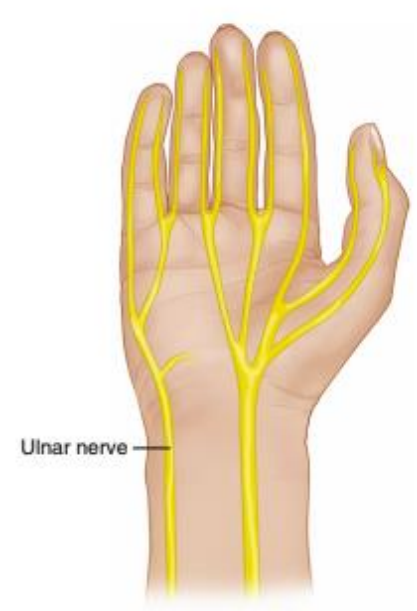

Imagen 2: localización del cubital. ${ }^{19}$

El nervio cubital puede anestesiarse tanto por volar como por dorsal, sin embargo su acceso más fácil y con menos riesgo es a nivel volar.

\section{Mediano}

- Qué se anestesia: Cara volar del 1, 2 y 3 dedos y mitad radial del 4 .

- Cómo se anestesia: Flexión muñeca, se identifica tendón palmar mayor y flexor radial del carpo, y se infiltra en el medio.

Cuidado con la aguja, vale la pena no inyectar tan profundamente para no lesionar el nervio. 

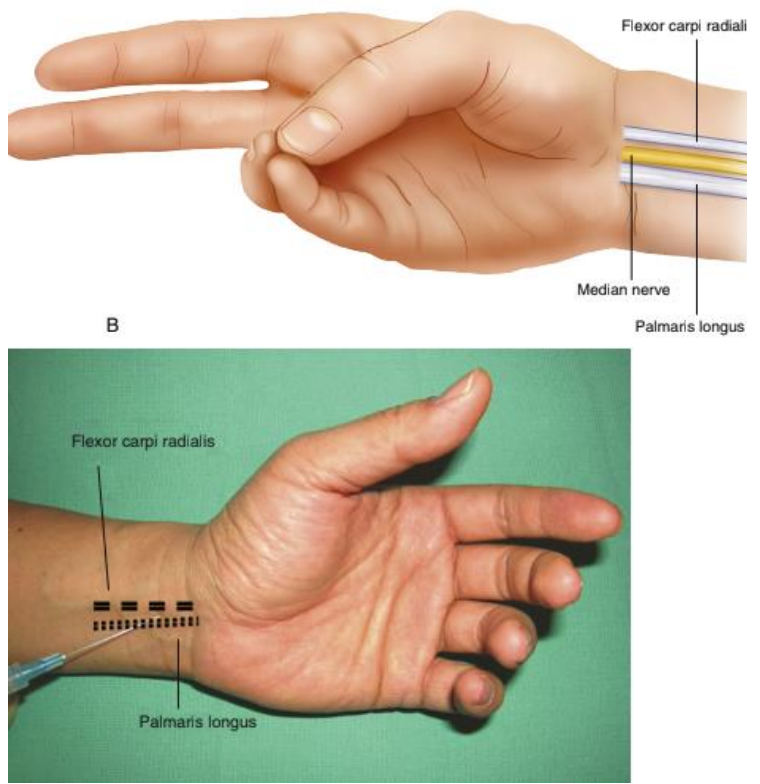

Imagen 3: localización del nervio mediano y anestesia del mismo. ${ }^{19}$

\section{Bloqueo intermetacarpiano}

- Qué se anestesia: se consigue mediante la inyección local del nervio anterior a su bifurcación una anestesia más completa a nivel distal.

- Cómo se anestesia: a nivel metacarpiano se inyecta de forma lateral anestésico local.
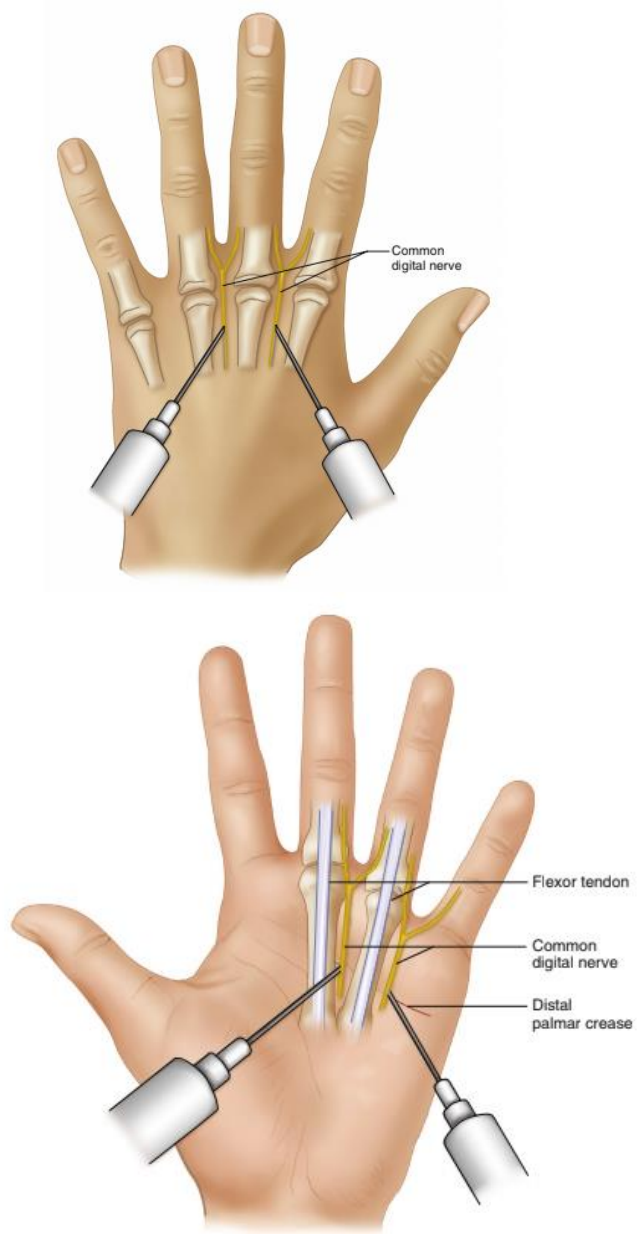

Imagen 3: bloqueo intermetacarpiano; técnica de anestesia. 19

\section{Bloqueo digital.}

Existen dos técnicas principales para la anestesia digital:

- Inyección única a nivel volar. A veces se queda sin anestesiar la parte dorsal distal del dedo. ${ }^{20,21}$

- Dos inyecciones a nivel dorsal. A veces se queda sin anestesiar la parte del pulpejo del dedo. ${ }^{20,21}$ 


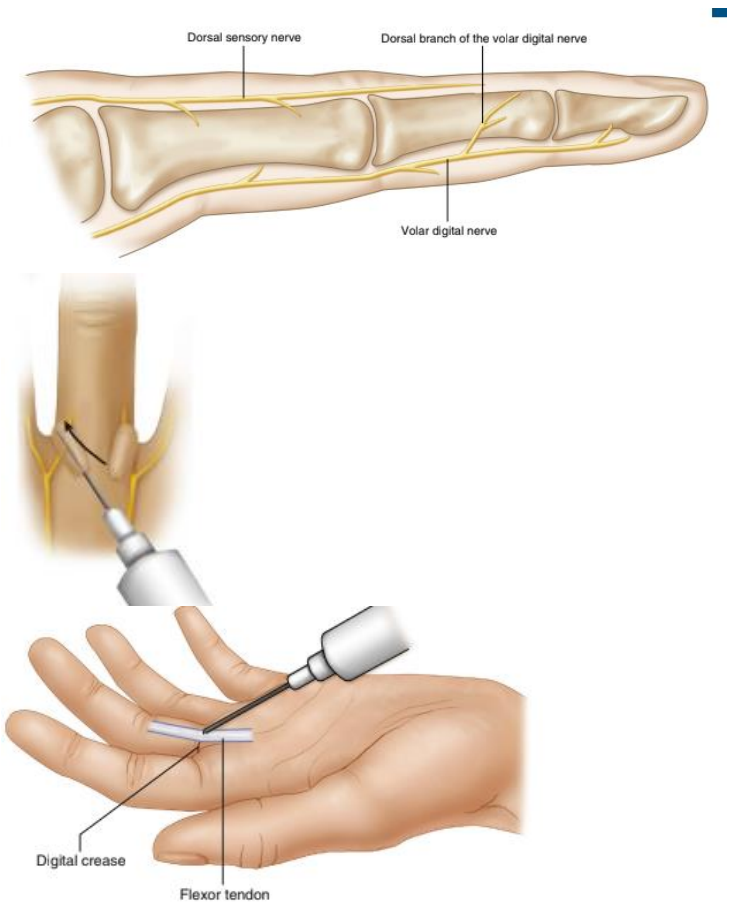

Imagen 4: bloqueo digital; inyección dorsal y volar. ${ }^{19}$

\section{¿Y SI NO USO ADRENALINA? TÉCNICAS DE ISQUEMIA}

Existen dos técnicas de isquemia muy utilizadas. La primera de ellas es utilizar un esfingomanómetro manual y mantenerlo a mayor presión que la arterial. De esta forma se consigue isquemia del miembro superior de forma temporal.

Otra de las técnicas ampliamente utilizadas a nivel de los dedos es utilizar un guante a modo de anillo de forma que produzca una isquemia distal. Cabe recordar que si se utiliza esta técnica será necesario colocar un mosquito para luego acordarnos siempre de retirar el guante. Si se dejara y encima aplicáramos un vendaje podríamos producir el mismo efecto que el de un anillo que no se puede sustraer en un dedo tumefacto.

\section{EXTREMIDAD INFERIOR}

El bloqueo de la extremidad inferior cada vez está teniendo mayores aplicaciones tanto en urgencias de traumatología como también en las cirugías del día a día.

\section{Bloqueo ramas terminales del tobillo}

\section{Peroneo superficial}

- Qué se anestesia: La región lateral distal de la pierna, el dorso del pie y los dedos del pie (salvo el primer espacio interdigital)

- Cómo se anestesia: Palpar el maléolo lateral e ir de forma proximal $8-10 \mathrm{~cm}$ anterior al borde de la diáfisis tibial

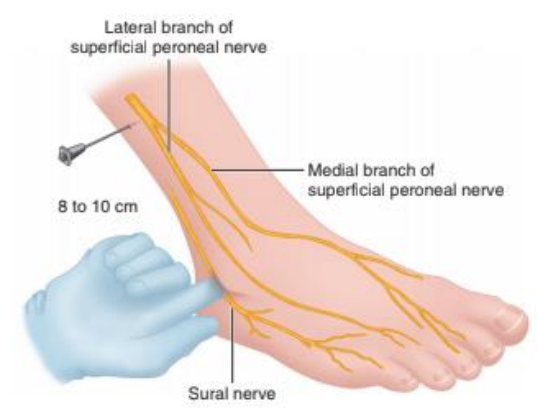

Imagen 5: localización del nervio peroneo superficial y anestesia del mismo. ${ }^{19}$

\section{Peroneo profundo}

- Qué se anestesia: El primer espacio interdigital.

- Cómo se anestesia: La arteria tibial anterior puede ser palpada a 4-5 $\mathrm{cm}$ de la articulación del tobillo. Se usa el tendón del tibial anterior, del extensor común de los dedos y del extensor del Hallux.

Si no se palpa la arteria, usar de referencia el tendón del tibial anterior $\mathrm{cm}$ e infiltrar lateral a $1-5 \mathrm{~cm}$ 


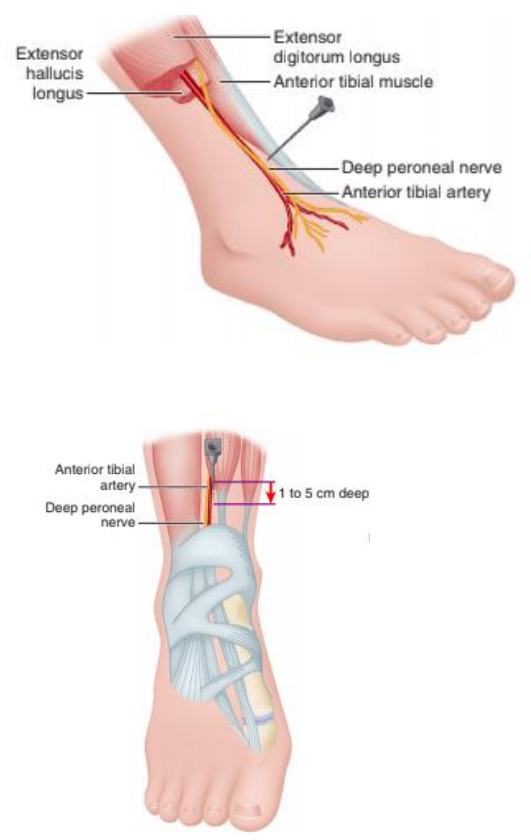

Imagen 6: localización del nervio peroneo profundo y anestesia del mismo. ${ }^{19}$

\section{Safeno}

- Qué se anestesia: Cara medial del pie.

- Cómo se anestesia: palpar el maléolo medial, y a 3-5 cm, se introduce la jeringuilla. El nervio safeno está medial a la vena safena

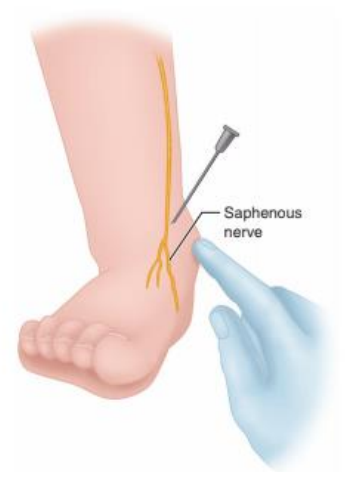

Imagen 7: localización del nervio safeno y anestesia del mismo. ${ }^{19}$

\section{4- Sural}

- Qué se anestesia: La cara lateral del pie.

- Cómo se anestesia: Palpar el maléolo lateral.A $5 \mathrm{~cm}$ proximal al maléolo se palpa el tendón del peroneo largo pegado al borde subcutáneo del peroné. A la mitad de la distancia entre el tendón y el Aquiles pasa el nervio sural.

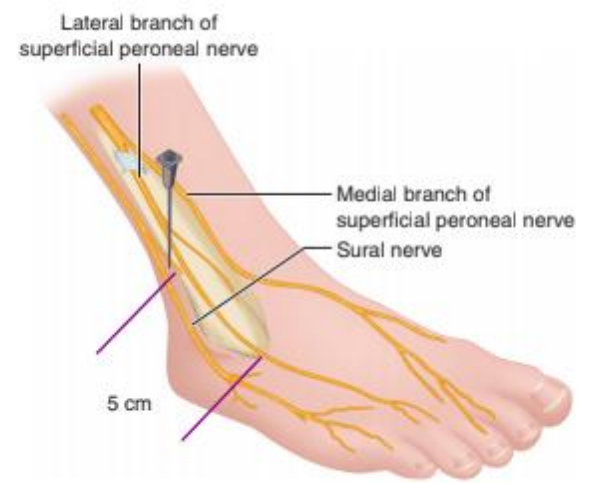

Imagen 8: localización del nervio sural y anestesia del mismo. ${ }^{19}$

\section{Tibial}

- Qué se anestesia: el talón, y por las dos ramas que da a posteriori; la parte medial de la planta, y los tres dedos y medio mediales $\left(1^{\circ}\right.$ a $\left.4^{\circ}\right)$, incluyendo las uñas, y la planta lateral, y un dedo y medio lateral $\left(4^{\circ}\right.$ y $\left.5^{\circ}\right)$.

- Cómo se anestesia: palpar el borde posteriomedial de la tibia, $5 \mathrm{~cm}$ proximal al maléolo medial. Se busca el flexor largo de los dedos y el tendón del tibial posterior. Luego se palpa el borde medial del tendón de Aquiles. A la mitad de la distancia entre ambos, pasa la arteria. 


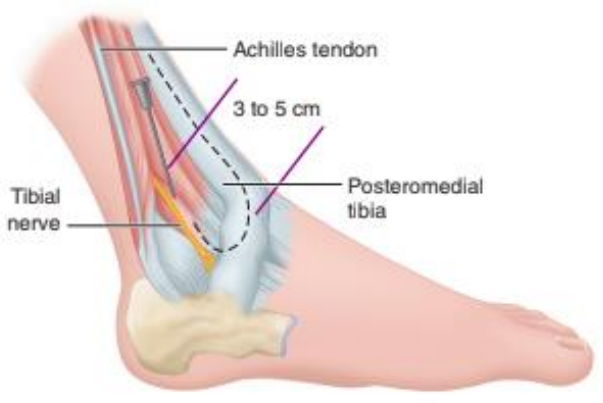

Imagen 9: localización del nervio tibial y anestesia del mismo. ${ }^{19}$

\section{Bloqueo digital}

En el bloqueo digital del pie se realizan las mismas técnicas que en el bloqueo digital de las manos.

\section{BIBLIOGRAFÍA}

1- Hagert E. Clinical diagnosis and wide-awake surgical treatment of proximal median nerve entrapment at the elbow: a prospective study. Hand (N Y). 2013 Mar;8(1):41-6

2- Denkler K. Dupuytren's fasciectomies in 60 consecutive digits using lidocaine with epinephrine and no tourniquet. PlastReconstrSurg. 2005 Mar;115(3):802-10

3- Farhangkhoee $\mathrm{H}$, Lalonde J, Lalonde $\mathrm{DH}$. Wide-awake trapeziectomy: video detailing local anesthetic injection and surgery. Hand (N Y). 2011 Dec;6(4):466-7

4- Matsuda T, Taniguchi A, Hayashi K, Nakanishi Y, Tanaka Y. Obtaining Adequate Tension for Extensor Hallucis Longus Tendon Rupture Repair Using Wide-Awake Surgery: A Case Report. J FootAnkleSurg. 2018 Mar - Apr;57(2):414-417

5- Wright J, MacNeill AL, Mayich DJ. A prospective comparison of wideawake local anesthesia and general anesthesia for forefoot surgery. FootAnkleSurg. 2019 Apr;25(2):211214
6- MacNeill AL, Mayich DJ. Wideawake foot and ankle surgery: A retrospective analysis. FootAnkleSurg. 2017 Dec;23(4):307310. doi:10.1016/j.fas.2016.09.004. Epub 2016 Oct 5

7- Lirk P, Picardi S, Hollmann MW. Local anaesthetics: 10 essentials. Eur J Anaesthesiol. 2014 Nov;31(11):575-85

8- Thomson CJ, Lalonde DH, Denkler KA, Feicht AJ. A critical look at the evidence for and against elective epinephrine use in the finger. PlastReconstrSurg. $\quad 2007$ Jan;119(1):260-6

9- Prabhakar $H$, Rath $S$, Kalaivani $M$, Bhanderi N. Adrenaline with lidocaine for digital nerve blocks. Cochrane DatabaseSyst Rev. 2015 Mar 19;(3)

10- Lalonde D, Eaton C, Amadio P, Jupiter J. Wide-awake Hand and Wrist Surgery: A New Horizon in Outpatient Surgery. InstrCourseLect. 2015;64:249-59

11- Muck AE, Bebarta VS, Borys DJ, Morgan DL. Six years of epinephrine digital injections: absence of significant local or systemic effects. Ann EmergMed. 2010 Sep;56(3):270-4

12- Lalonde $D$, Bell $M$, Benoit $P$, Sparkes G, Denkler K, Chang P. A multicenter prospective study of 3,110 consecutive cases of elective epinephrine use in the fingers and hand: the Dalhousie Project clinical phase. J Hand Surg Am. 2005 Sep;30(5):1061-7

13- Chowdhry S, Seidenstricker L, Cooney DS, Hazani R, Wilhelmi BJ. Do not use epinephrine in digital blocks: myth or truth? Part II. A retrospective review of 1111 cases. PlastReconstr Surg. 2010 Dec;126(6):2031-4

14- Nodwell T, Lalonde D. How long does it take phentolamine to reverse adrenaline-induced vasoconstriction in the finger and hand? A prospective,randomized, 
blinded study: The Dalhousie project experimental phase. Can J Plast Surg. 2003 Winter;11(4):18790

15- Quaba O, Huntley JS, Bahia $H_{\text {, }}$ McKeown DW. A users guide for reducing the pain of local anaesthetic administration. EmergMed J. 2005 Mar;22(3):188-9

16- Kouba DJ, LoPiccolo MC, Alam M, Bordeaux JS, Cohen B, Hanke CW, JellinekN,Maibach HI, Tanner JW, Vashi N, Gross KG, Adamson T, Begolka WS, MoyanoJV.Guidelines for the use of local anesthesia in office-based dermatologic surgery. J Am AcadDermatol. 2016 Jun;74(6):1201-19

17- Drugs.com [Internet]. Lidocaine information from drugs.com; c1996-2018 [Updated: 12 June 2018].

Availablefrom: https://www.drugs.c $\mathrm{om} / \mathrm{mtm} /$ lidocaine-injection.html

18- Drugs.com [Internet]. Mepivacaine information from drugs.com; c1996-2018 [Updated: 13 May 2019]. Available from: https://www.drugs.com/mtm/ mepivacaine.html

19- Canale ST, Beaty J. Campbell's Operative Orthopaedics, 12th ed. Mosby; 2012.

20- Williams JG, Lalonde DH. Randomized comparison of the single-injection volar subcutaneous block and the two-injection dorsal block for digital anesthesia. PlastReconstr Surg. 2006 Oct;118(5):1195-200

21- Wheelock ME, Leblanc $M$, Chung $B$, Williams J, Lalonde $\mathrm{DH}$. Is it true that injecting palmar finger skin hurts more than dorsal skin? New level 1 evidence.Hand (N Y). 2011 Mar;6(1):47-9. doi: 10.1007/s11552010-9288-2. Epub 2010 Sep 11 